\begin{tabular}{|c|c|c|}
\hline \multicolumn{3}{|c|}{ Survey Information } \\
\hline Location and Date & Response & Code \\
\hline Unique ID (Cluster (CEB/Village) ID and Patient ID) & ----- & 1 \\
\hline Cluster (CEB/Village) name & & 12 \\
\hline Interviewer ID & $\mathrm{L} \perp$ & 12 \\
\hline Date of completion of the instrument & 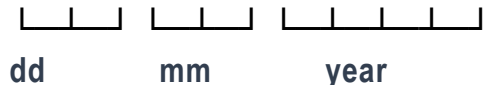 & 14 \\
\hline
\end{tabular}

\begin{tabular}{|c|c|c|c|c|}
\hline Consent, Interview Language and Name & \multicolumn{3}{|c|}{ Response } & \multirow{2}{*}{$\begin{array}{c}\text { Code } \\
15\end{array}$} \\
\hline Consent has been read and obtained & $\begin{array}{l}\text { Yes } \\
\text { No }\end{array}$ & 2 & 1 & \\
\hline Interview Language & $\begin{array}{r}\text { English } \\
\text { Hindi } \\
\text { Punjabi } \\
\text { Other }\end{array}$ & & $\begin{array}{l}1 \\
2 \\
3 \\
4 \\
\end{array}$ & 16 \\
\hline $\begin{array}{l}\text { Time of interview } \\
\text { (24 hour clock) }\end{array}$ & & & لـ & 17 \\
\hline Family Surname & & & & 18 \\
\hline First Name & & & & 19 \\
\hline Contact phone number where possible & & & & 110 \\
\hline Unique Identification Number & & & & 111 \\
\hline
\end{tabular}




\section{Demographic information}

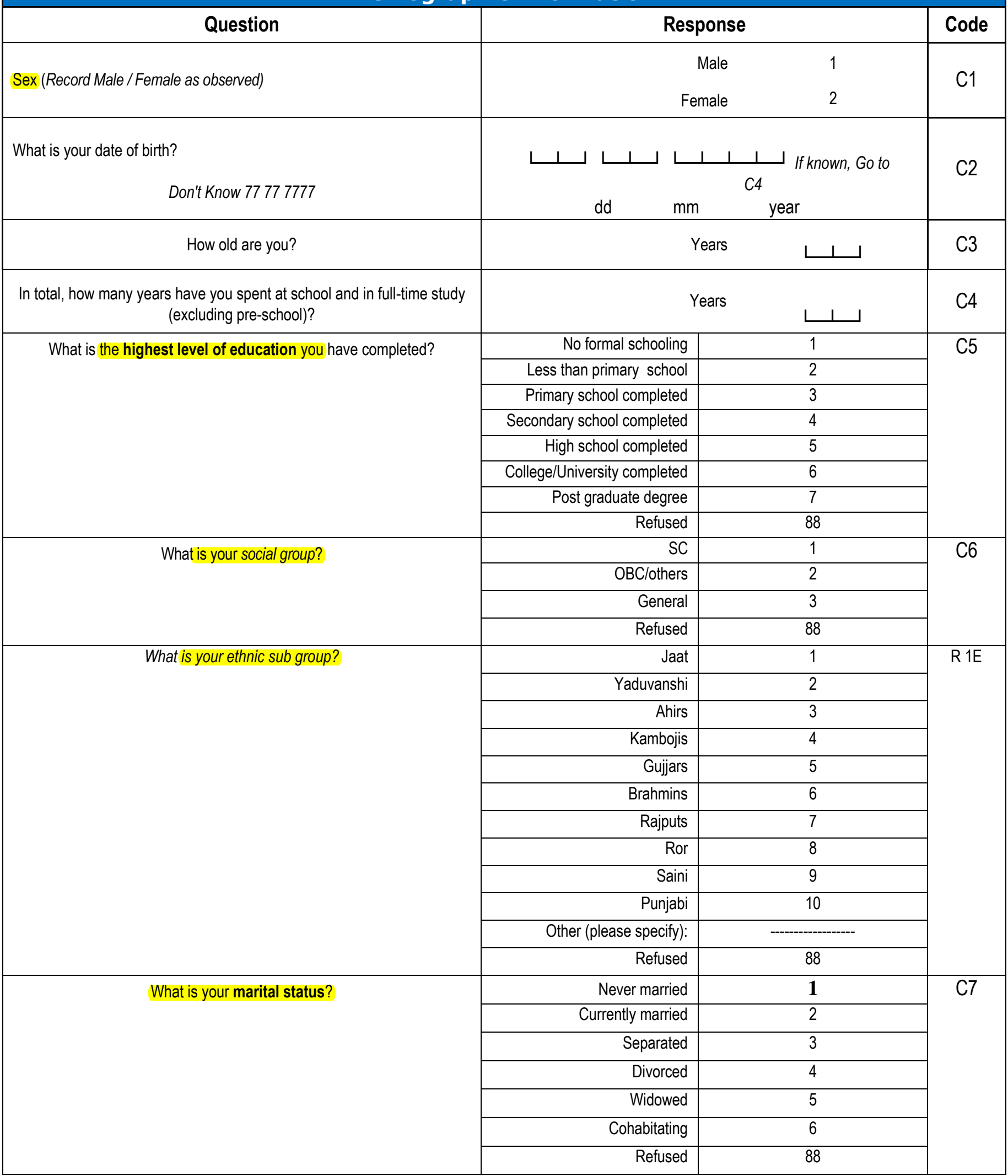




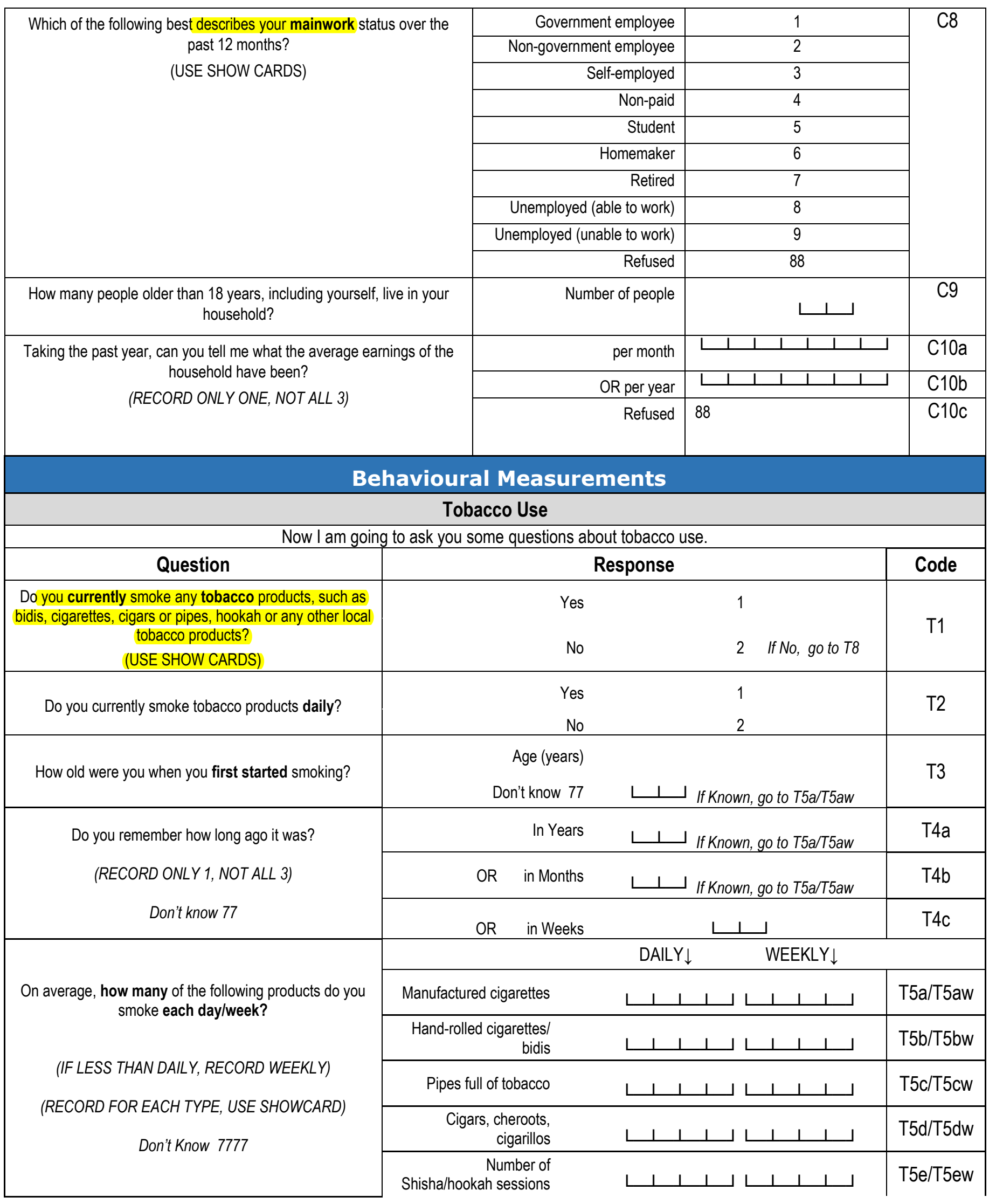




\begin{tabular}{|c|c|c|c|}
\hline & Other & 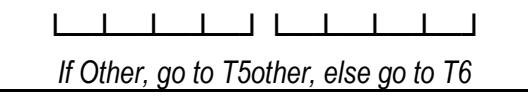 & T5f/T5fw \\
\hline & Other (please specify): & لـ & $\begin{array}{l}\text { T5other/ } \\
\text { T5otherw }\end{array}$ \\
\hline $\begin{array}{l}\text { During the past } 12 \text { months, have you tried to stop } \\
\text { smoking? }\end{array}$ & $\begin{array}{r}\text { Yes } \\
\text { No }\end{array}$ & $\begin{array}{l}1 \\
2\end{array}$ & T6 \\
\hline $\begin{array}{l}\text { During any visit to a doctor or other health worker in the } \\
\text { past } 12 \text { months, were you advised to quit smoking } \\
\text { tobacco? }\end{array}$ & $\begin{array}{r}\text { Yes } \\
\text { No } \\
\text { No visit during the past 12 } \\
\text { months }\end{array}$ & $\begin{array}{l}1 \text { If } T 2=Y e s, \text { go to } T 12 \text {; if } T 2=N o \text {, goto } T 9 \\
2 \text { If } T 2=Y e s \text {, go to } T 12 \text {; if } T 2=N o \text {, go to } T 9 \\
3 \text { If } T 2=Y e s \text {, go to } T 12 \text {; if } T 2=N o \text {, go to } T 9\end{array}$ & T7 \\
\hline $\begin{array}{l}\text { In the past, did you eversmoke any tobacco products? } \\
\text { (USE SHOWCARD) }\end{array}$ & $\begin{array}{l}\text { Yes } \\
\text { No }\end{array}$ & 2 If No, go to T12 & T8 \\
\hline In the past, did you ever smoke daily? & $\begin{array}{l}\text { Yes } \\
\text { No }\end{array}$ & $\begin{array}{l}1 \text { If } T 1=Y e s, \text { go to } T 12 \text {, else go to } T 10 \\
\text { 2lf } T 1=Y e s \text {, go to } T 12 \text {, else go to } T 10\end{array}$ & T9 \\
\hline
\end{tabular}

\begin{tabular}{|c|c|c|c|}
\hline \multirow[t]{2}{*}{ How old were you when you stopped smoking? } & Age (years) & \multirow{2}{*}{ ᄂــــ If Known, go to T12 } & \multirow[t]{2}{*}{ T10 } \\
\hline & Don't Know 77 & & \\
\hline $\begin{array}{l}\text { How long ago did you stop smoking? } \\
\text { (RECORD ONLY 1, NOT ALL 3) }\end{array}$ & Years ago & ᄂلــلIf Known, go to T12 & T11a \\
\hline (RECORD ONLY 1, NOT ALL 3) & OR Months ago & ـــــ If Known, go to T12 & $\mathrm{T} 11 \mathrm{~b}$ \\
\hline Don't Know 77 & Weeks ago & لــ & T11c \\
\hline \multirow{2}{*}{$\begin{array}{l}\text { Do you currently use any smokeless tobacco } \\
\text { products such as [snuff, chewing tobacco, tuibu, } \\
\text { gutka, betel, Pan masala]? (USE SHOWCARD) }\end{array}$} & Yes & 1 & \multirow[t]{2}{*}{ T12 } \\
\hline & No & 2 If No, go to T15 & \\
\hline \multirow{2}{*}{$\begin{array}{l}\text { Do you currently usesmokeless tobacco products } \\
\text { daily? }\end{array}$} & Yes & 1 & \multirow{2}{*}{ T13 } \\
\hline & No & If No, go to T14aw & \\
\hline \multirow[b]{3}{*}{$\begin{array}{l}\text { (IF LESS THAN DAILY, RECORD WEEKLY) } \\
\text { (RECORD FOR EACH TYPE USE SHOWCARD) }\end{array}$} & \multicolumn{3}{|c|}{ DAILY $\downarrow \quad$ WEEKLY $\downarrow$} \\
\hline & Tobacco Snuff, by mouth & 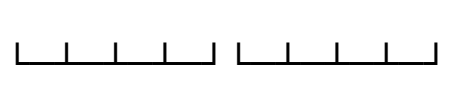 & $\begin{array}{l}\text { T14al } \\
\text { T14aw }\end{array}$ \\
\hline & Snuff, by nose & 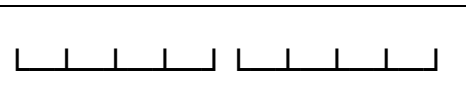 & $\begin{array}{l}\text { T14b/ } \\
\text { T14bw }\end{array}$ \\
\hline \multirow{4}{*}{ Don't Know 7777} & Chewing tobacco & لـ & $\begin{array}{l}\text { T14c/ } \\
\text { T14cw }\end{array}$ \\
\hline & Betel, quid with tobacco & 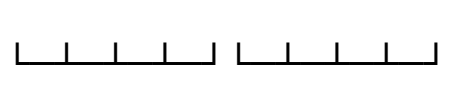 & $\begin{array}{l}\mathrm{T} 14 \mathrm{~d} / \\
\mathrm{T} 14 \mathrm{dw}\end{array}$ \\
\hline & Other & 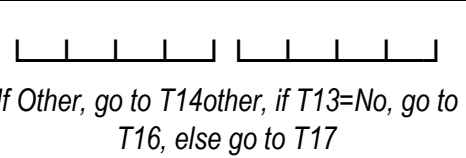 & $\begin{array}{l}\text { T14e/ } \\
\text { T14ew }\end{array}$ \\
\hline & Other (please specify): & 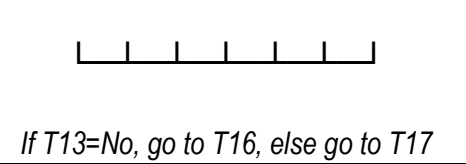 & $\begin{array}{l}\text { T140ther/ } \\
\text { T14otherw }\end{array}$ \\
\hline
\end{tabular}




\begin{tabular}{|c|c|c|c|}
\hline $\begin{array}{l}\text { In the past, did you ever use smokeless tobacco } \\
\text { products such as [snuff, chewing tobacco, gutka or } \\
\text { betel with tobacco, pan masala]? }\end{array}$ & Yes & 1 & T15 \\
\hline \multirow{2}{*}{$\begin{array}{c}\text { In the past, did you ever use smokeless tobacco } \\
\text { products such as [snuff, chewing tobacco, gutka,betel } \\
\text { with tobacco daily? }\end{array}$} & Yes & 1 & \multirow[t]{2}{*}{ T16 } \\
\hline & No & 2 & \\
\hline $\begin{array}{l}\text { During the past } 30 \text { days, did someone smoke in your } \\
\text { home? }\end{array}$ & Yes & 1 & T17 \\
\hline \multirow{3}{*}{$\begin{array}{l}\text { During the past } 30 \text { days, did someone smoke in } \\
\text { closed areas in your workplace (in the building, in a } \\
\text { work area or a specific office)? }\end{array}$} & Yes & 1 & \multirow[t]{3}{*}{ T18 } \\
\hline & No & 2 & \\
\hline & Don't work in a closed area & 3 & \\
\hline
\end{tabular}




\begin{tabular}{|c|c|c|c|}
\hline \multicolumn{4}{|c|}{ Alcohol Consumption } \\
\hline \multicolumn{4}{|c|}{ The next questions ask about the consumption of alcohol. } \\
\hline Question & \multicolumn{2}{|c|}{ Response } & Code \\
\hline \multirow{2}{*}{$\begin{array}{c}\text { Have you ever consumed any alcohol such as beer, wine, } \\
\text { whisky spirits or locally prepared alcohol? } \\
\text { (USE SHOWCARD OR SHOW EXAMPLES) }\end{array}$} & Yes & 1 & \multirow[t]{2}{*}{ A1 } \\
\hline & No & 2 If No, go to A16 & \\
\hline \multirow[t]{2}{*}{ Have you consumed any alcohol within the past 12 months? } & Yes & 1 If Yes, go to A4 & \multirow[t]{2}{*}{ A2 } \\
\hline & No & 2 & \\
\hline \multirow{2}{*}{$\begin{array}{l}\text { Have you stopped drinking due to health reasons, such as a } \\
\text { negative impact on your health or on the advice of your doctor or } \\
\text { other health worker? }\end{array}$} & Yes & 1 If Yes, go to $A 16$ & \multirow[t]{2}{*}{ A3 } \\
\hline & No & 2 If No, go to $A 16$ & \\
\hline \multirow{6}{*}{$\begin{array}{c}\text { During the past } 12 \text { months, how frequently have you had at } \\
\text { least one standard alcoholic drink? }\end{array}$} & Daily & 1 & \multirow[t]{6}{*}{ A4 } \\
\hline & 5-6 days per week & 2 & \\
\hline & 3-4 days per week & 3 & \\
\hline & 1-2 days per week & 4 & \\
\hline & $1-3$ days per month & 5 & \\
\hline & Less than once a month & 6 & \\
\hline \multirow[t]{2}{*}{ Have you consumed any alcohol within the past 30 days? } & Yes & 1 & \multirow[t]{2}{*}{ A5 } \\
\hline & No & 2 If No, go to $A 13$ & \\
\hline $\begin{array}{c}\text { During the past } 30 \text { days, on how many occasions did you have } \\
\text { at least one standard alcoholic drink? }\end{array}$ & $\begin{array}{r}\text { Number } \\
\text { Don't know } 77\end{array}$ & L」 & A6 \\
\hline $\begin{array}{l}\text { During the past } 30 \text { days, when you drank alcohol, how many } \\
\text { standarddrinks on average did you have during one drinking } \\
\text { occasion? } \\
\text { (USE SHOWCARD) }\end{array}$ & $\begin{array}{r}\text { Number } \\
\text { Don't know } 77\end{array}$ & Lلـ لــ & A7 \\
\hline $\begin{array}{c}\text { During the past } 30 \text { days, what was the largest number of } \\
\text { standard drinks you had on a single occasion, counting all types } \\
\text { of alcoholic drinks together? }\end{array}$ & $\begin{array}{l}\text { Largest number } \\
\text { Don't Know } 77\end{array}$ & 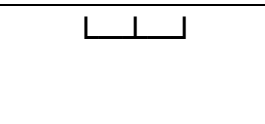 & $A 8$ \\
\hline $\begin{array}{l}\text { During the past } 30 \text { days, how many times did you have } \\
\text { six or more standard drinks in a single drinking occasion? }\end{array}$ & $\begin{array}{l}\text { Number of times } \\
\text { Don't Know } 77\end{array}$ & لـــل & $\mathrm{A} 9$ \\
\hline \multirow[t]{7}{*}{$\begin{array}{l}\text { During each of the past } 7 \text { days, how many standard drinks did } \\
\text { you have each day? }\end{array}$} & Monday & لــــــا & A10a \\
\hline & Tuesday & 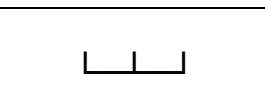 & $A 10 b$ \\
\hline & Wednesday & لـــــا & $A 10 c$ \\
\hline & Thursday & لــــــا & A10d \\
\hline & Friday & لــــــا & A10e \\
\hline & Saturday & لـــــــــــــ & A10f \\
\hline & Sunday & لـــــا & $\mathrm{A} 10 \mathrm{~g}$ \\
\hline
\end{tabular}


I have just asked you about your consumption of alcohol during the past 7 days. The questions were about alcohol in general, while the next questions refer to your consumption of homebrewed alcohol, alcohol brought over the border/from another country, any alcohol not intended for drinking or other untaxed alcohol. Please only think about these types of alcohol when answering the next questions.

\begin{tabular}{|c|c|c|c|}
\hline Question & Response & & Code \\
\hline $\begin{array}{l}\text { During the past } 7 \text { days, did you consume any homebrewed } \\
\text { alcohol, any alcohol brought over the border/from another } \\
\text { country, any alcohol not intended for drinking or other } \\
\text { untaxed alcohol? } \\
\text { (USE SHOWCARD) }\end{array}$ & No & 2 If No, go to A13 & A11 \\
\hline \multirow{3}{*}{$\begin{array}{l}\text { On average, how many standard drinks of the following did } \\
\text { you consume during the past } 7 \text { days? }\end{array}$} & $\begin{array}{r}\text { DesiSharab (santra, khatta, Malwa) or } \\
\text { Homebrewed spirits }\end{array}$ & 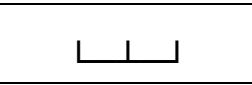 & A12a \\
\hline & & & \\
\hline & $\begin{array}{r}\begin{array}{r}\text { Homebrewed beer or wine, e.g. beer, or } \\
\text { fruit wine }\end{array} \\
\end{array}$ & $\downarrow$ & $A 12 b$ \\
\hline \multirow[t]{3}{*}{ (USE SHOWCARD) } & $\begin{array}{r}\text { Alcohol brought over the border/from } \\
\text { another country }\end{array}$ & $\perp$ & $\mathrm{A} 12 \mathrm{C}$ \\
\hline & $\begin{array}{r}\text { Alcohol not intended for drinking, e.g. } \\
\text { alcohol-based medicines, perfumes, after } \\
\text { shaves }\end{array}$ & \llcorner & $A 12 d$ \\
\hline & Other untaxed alcohol in the country & 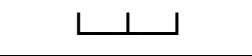 & $\mathrm{A} 12 \mathrm{e}$ \\
\hline \multirow{5}{*}{$\begin{array}{l}\text { During the past } 12 \text { months, how often have you found that you } \\
\text { were not able to stop drinking once you had started? }\end{array}$} & Daily or almost daily & 1 & \multirow{5}{*}{ A13 } \\
\hline & Weekly & 2 & \\
\hline & Monthly & 3 & \\
\hline & Less than monthly & 4 & \\
\hline & Never & 5 & \\
\hline \multirow{5}{*}{$\begin{array}{l}\text { During the past } 12 \text { months, how often have you failed to do } \\
\text { what was normally expected from you because of drinking? }\end{array}$} & Daily or almost daily & 1 & \multirow[t]{5}{*}{ A14 } \\
\hline & Weekly & 2 & \\
\hline & Monthly & 3 & \\
\hline & Less than monthly & 4 & \\
\hline & Never & 5 & \\
\hline \multirow{5}{*}{$\begin{array}{l}\text { During the past } 12 \text { months, how often have you needed a first } \\
\text { drink in the morning to get yourself going after a heavy drinking } \\
\text { session? }\end{array}$} & Daily or almost daily & 1 & \multirow[t]{5}{*}{ A15 } \\
\hline & Weekly & 2 & \\
\hline & Monthly & 3 & \\
\hline & Less than monthly & 4 & \\
\hline & Never & 5 & \\
\hline \multirow{5}{*}{$\begin{array}{l}\text { During the past } 12 \text { months, have you had family problems or } \\
\text { problems with your partner due to someone else's drinking? }\end{array}$} & Yes, more than monthly & 1 & \multirow[t]{5}{*}{ A16 } \\
\hline & Yes, monthly & 2 & \\
\hline & Yes, several times but less than monthly & 3 & \\
\hline & Yes, once or twice & 4 & \\
\hline & No & 5 & \\
\hline
\end{tabular}




\section{Diet}

The next questions ask about the fruits and vegetables that you usually eat. I have a nutrition card here that shows you some examples of local fruits and vegetables. Each picture represents the size of a serving. As you answer these questions please think of a typical week in the last year.

\begin{tabular}{|c|c|c|}
\hline Question & Response & Code \\
\hline $\begin{array}{c}\text { In a typical week, on how many days do you eat fruit? } \\
\text { (USE SHOWCARD) }\end{array}$ & $\begin{array}{c}\text { Number of days } \\
\text { Don't Know 77 }\end{array}$ & D1 \\
\hline $\begin{array}{c}\text { How many servings of fruit do you eat on one of those } \\
\text { days? (USE SHOWCARD) }\end{array}$ & $\begin{array}{c}\text { Number of servings } \\
\text { Don't Know 77 }\end{array}$ & D2 \\
\hline $\begin{array}{c}\text { In a typical week, on how many days do you eat } \\
\text { vegetables? (USE SHOWCARD) }\end{array}$ & $\begin{array}{c}\text { Number of days } \\
\text { Don't Know 77 }\end{array}$ & D3 to D3 \\
\hline $\begin{array}{c}\text { How many servings of vegetables do you eat on one of } \\
\text { those days? (USE SHOWCARD) }\end{array}$ & $\begin{array}{c}\text { Number of servings } \\
\text { Don't know 77 }\end{array}$ & D4 \\
\hline
\end{tabular}

\section{Dietary Salt}

With the next questions, we would like to learn more about salt in your diet. Dietary salt includes ordinary table salt, unrefined salt such as sea salt, iodized salt, and powders, and salty sauces such as soya sauce or fish sauce (see showcard). The following questions are on adding salt to the food right before you eat it, on how food is prepared in your home, on eating processed foods that are high in salt such as pickles, chutneys, and questions on controlling your salt intake. Please answer the questions even if you consider yourself to eat a diet low in salt.

\begin{tabular}{|c|c|c|c|}
\hline Question & Response & & Code \\
\hline \multirow{2}{*}{$\begin{array}{l}\text { How often do you add salt or a salty sauce such as soya } \\
\text { sauce to your food right before you eat it or as you are eating it? }\end{array}$} & Always & 1 & \multirow{6}{*}{ D5 } \\
\hline & Often & 2 & \\
\hline \multirow{4}{*}{$\begin{array}{l}\text { (SELECT ONLY ONE) } \\
\text { (USE SHOWCARD) }\end{array}$} & Sometimes & 3 & \\
\hline & Rarely & 4 & \\
\hline & Never & 5 & \\
\hline & Don't know & 77 & \\
\hline \multirow{6}{*}{$\begin{array}{l}\text { How often is salt, salty seasoning or a salty sauce added in } \\
\text { cooking or preparing foods in your household? }\end{array}$} & Always & 1 & \multirow{6}{*}{ D6 } \\
\hline & Often & 2 & \\
\hline & Sometimes & 3 & \\
\hline & Rarely & 4 & \\
\hline & Never & 5 & \\
\hline & Don't know & 77 & \\
\hline \multirow{6}{*}{$\begin{array}{c}\text { How often do you eat processed food high in salt? By } \\
\text { processed food high in salt, I mean foods that have been altered } \\
\text { from their natural state, such as packaged salty snacks, canned } \\
\text { salty food, salty food prepared at a fast food restaurant, pickles, } \\
\text { chutneys }\end{array}$} & Always & 1 & \multirow{6}{*}{ D7 } \\
\hline & Often & 2 & \\
\hline & Sometimes & 3 & \\
\hline & Rarely & 4 & \\
\hline & Never & 5 & \\
\hline & Don't know & 77 & \\
\hline \multirow{4}{*}{ How much salt or salty sauce do you think you consume? } & Far too much & 1 & \multirow{4}{*}{ D8 } \\
\hline & Too much & 2 & \\
\hline & Just the right amount & 3 & \\
\hline & Too little & 4 & \\
\hline
\end{tabular}




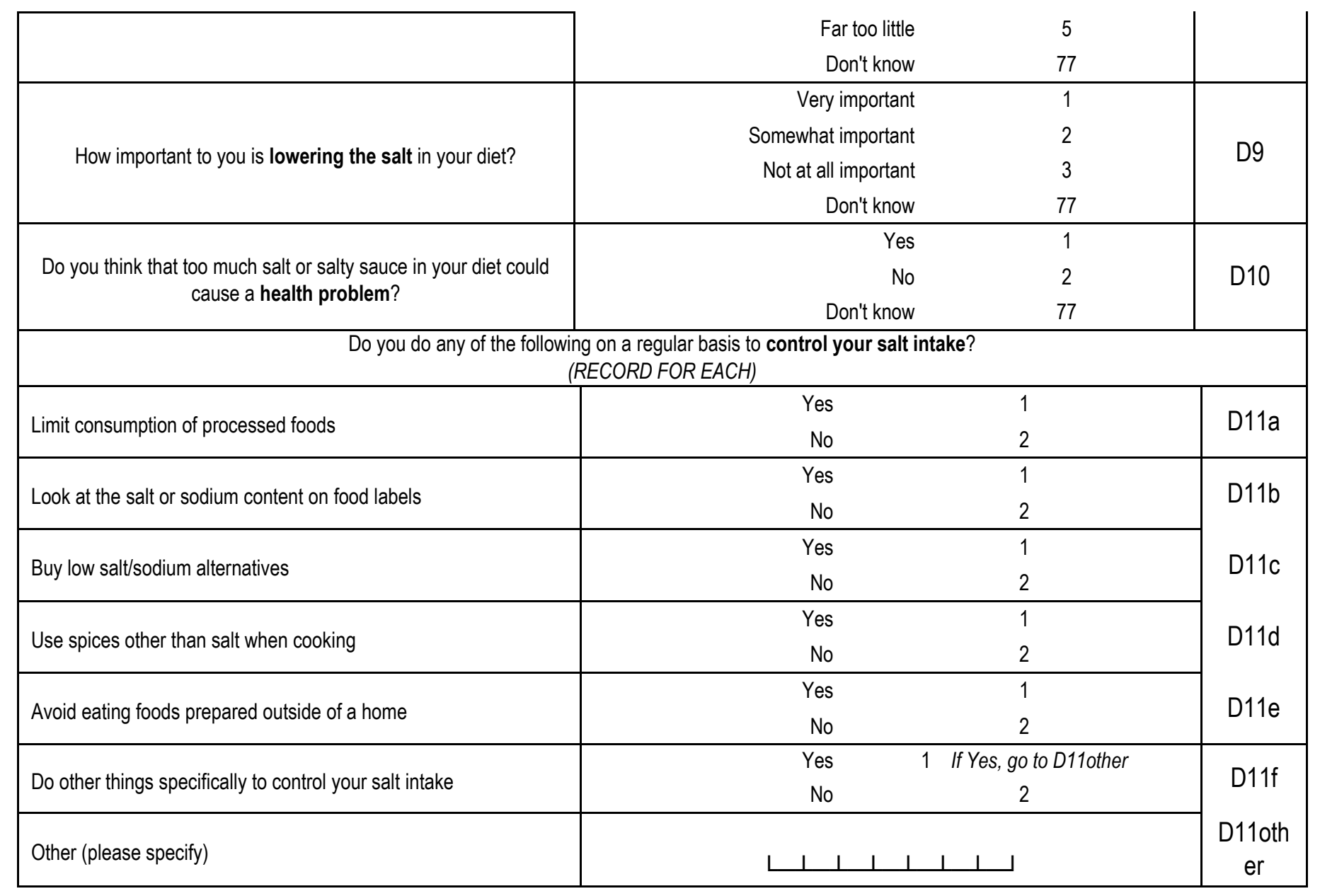

\begin{tabular}{|c|c|c|c|}
\hline \multirow{9}{*}{$\begin{array}{l}\text { What type of oil or fat is most often used for meal preparation in } \\
\text { your household? } \\
\text { (USE SHOWCARD) }\end{array}$} & Mustard oil & 1 & \multirow{8}{*}{ D12 } \\
\hline & Soyabean oil & 2 & \\
\hline & Butter or Pure ghee & 3 & \\
\hline & Sunflower oil & 4 & \\
\hline & Other & 5 If Other, go to D12 other & \\
\hline & None in particular & 6 & \\
\hline & None used & 7 & \\
\hline & Don't know & 77 & \\
\hline & Other & $ل$ & D12other \\
\hline $\begin{array}{l}\text { On average, how many meals per week do you eat that were not } \\
\text { prepared at a home? By meal, I mean breakfast, lunch and } \\
\text { dinner. }\end{array}$ & $\begin{array}{r}\text { Number } \\
\text { Don't know } 77\end{array}$ & Lلـ لــ & D13 \\
\hline
\end{tabular}




\section{Physical Activity}

Next I am going to ask you about the time you spend doing different types of physical activity in a typical week. Please answer these questions even if you do not consider yourself to be a physically active person.

Think first about the time you spend doing work. Think of work as the things that you have to do such as paid or unpaid work, study/training, household chores, harvesting food/crops, fishing or hunting for food, seeking employment.In answering the following questions 'vigorousintensity activities' are activities that require hard physical effort and cause large increases in breathing or heart rate, 'moderate-intensity activities' are activities that require moderate physical effort and cause small increases in breathing or heart rate.

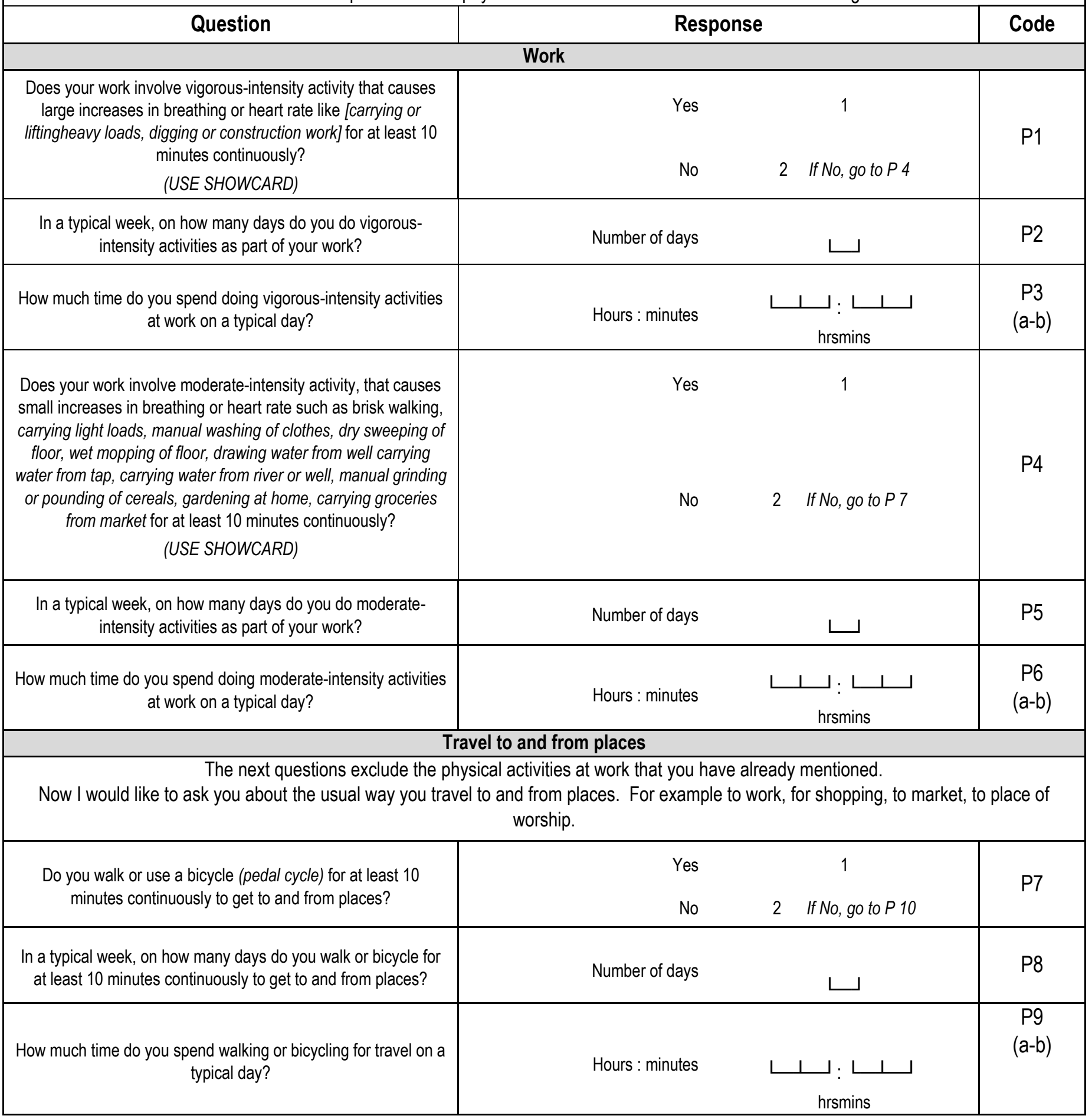




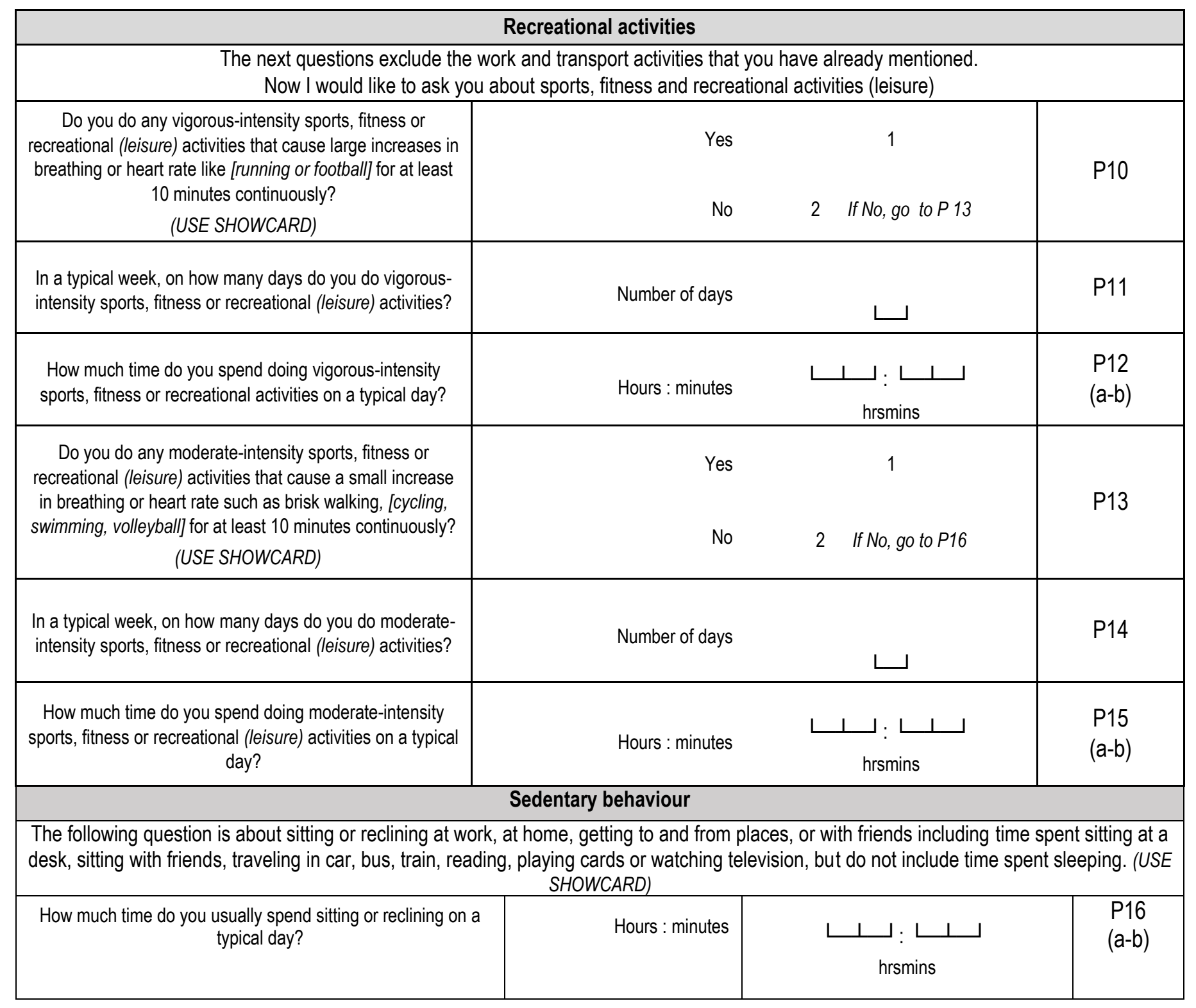

\begin{tabular}{|c|c|c|c|c|}
\hline \multicolumn{5}{|c|}{ History of Raised Blood Pressure } \\
\hline Question & \multicolumn{3}{|c|}{ Response } & Code \\
\hline \multirow{2}{*}{$\begin{array}{l}\text { Have you ever had your blood pressure measured by a doctor } \\
\text { or other health worker? }\end{array}$} & Yes & & 1 & \multirow{2}{*}{$\mathrm{H} 1$} \\
\hline & No & 2 & If No, go to $\mathrm{H} 6$ & \\
\hline \multirow{2}{*}{$\begin{array}{l}\text { Have you ever been told by a doctor or other health worker that } \\
\text { you have raised blood pressure or hypertension? }\end{array}$} & Yes & & 1 & \multirow{2}{*}{$\mathrm{H} 2 \mathrm{a}$} \\
\hline & No & 2 & If No, go to $\mathrm{H} 6$ & \\
\hline \multirow{2}{*}{ Have you been told in the past 12 months? } & Yes & & 1 & \multirow{2}{*}{$\mathrm{H} 2 \mathrm{~b}$} \\
\hline & No & & 2 & \\
\hline \multirow{2}{*}{$\begin{array}{l}\text { In the past two weeks, have you taken any drugs (medication) } \\
\text { for raised blood pressure prescribed by a doctor or other health } \\
\text { worker? }\end{array}$} & Yes & & 1 & \multirow{2}{*}{$\mathrm{H} 3$} \\
\hline & No & & 2 & \\
\hline
\end{tabular}




\begin{tabular}{|c|c|c|}
\hline $\begin{array}{c}\text { Have you ever seen a traditional healer for raised blood } \\
\text { pressure or hypertension? }\end{array}$ & Yes & 1 \\
\hline $\begin{array}{c}\text { No } \\
\text { Are you currently taking any herbal or traditional remedy for your } \\
\text { raised blood pressure? }\end{array}$ & Yes & 1 \\
\hline
\end{tabular}

\begin{tabular}{|c|c|c|c|c|}
\hline \multicolumn{5}{|c|}{ History of Diabetes } \\
\hline $\begin{array}{l}\text { Have you ever had your blood sugar measured by a doctor or } \\
\text { other health worker? }\end{array}$ & $\begin{array}{l}\text { Yes } \\
\text { No }\end{array}$ & 2 & $\begin{array}{c}1 \\
\text { If No, go to } H 12\end{array}$ & $\mathrm{H} 6$ \\
\hline $\begin{array}{l}\text { Have you ever been told by a doctor or other health worker that } \\
\text { you have raised blood sugar or diabetes? }\end{array}$ & $\begin{array}{l}\text { Yes } \\
\text { No }\end{array}$ & 2 & $\begin{array}{c}1 \\
\text { If No, go to } H 12\end{array}$ & $\mathrm{H} 7 \mathrm{a}$ \\
\hline Have you been told in the past 12 months? & $\begin{array}{l}\text { Yes } \\
\text { No }\end{array}$ & & $\begin{array}{l}1 \\
2\end{array}$ & $\mathrm{H} 7 \mathrm{~b}$ \\
\hline $\begin{array}{l}\text { In the past two weeks, have you taken any drugs (medication) } \\
\text { for diabetes prescribed by a doctor or other health worker? }\end{array}$ & $\begin{array}{l}\text { Yes } \\
\text { No }\end{array}$ & & $\begin{array}{l}1 \\
2\end{array}$ & H8 \\
\hline $\begin{array}{l}\text { Are you currently taking insulin for diabetes prescribed by a } \\
\text { doctor or other health worker? }\end{array}$ & $\begin{array}{l}\text { Yes } \\
\text { No }\end{array}$ & & $\begin{array}{l}1 \\
2\end{array}$ & $\mathrm{H} 9$ \\
\hline $\begin{array}{l}\text { Have you ever seen a traditional healer for diabetes or raised } \\
\text { blood sugar? }\end{array}$ & $\begin{array}{l}\text { Yes } \\
\text { No }\end{array}$ & & $\begin{array}{l}1 \\
2\end{array}$ & $\mathrm{H} 10$ \\
\hline $\begin{array}{l}\text { Are you currently taking any herbal or traditional remedy for your } \\
\text { diabetes? }\end{array}$ & $\begin{array}{l}\text { Yes } \\
\text { No }\end{array}$ & & $\begin{array}{l}1 \\
2\end{array}$ & $\mathrm{H} 11$ \\
\hline
\end{tabular}

\begin{tabular}{|c|c|c|c|c|}
\hline \multicolumn{5}{|c|}{ History of Raised Total Cholesterol } \\
\hline Question & Respo & & & Code \\
\hline \multirow{2}{*}{$\begin{array}{l}\text { Have you ever had your cholesterol (fat levels in your blood) } \\
\text { measured by a doctor or other health worker? }\end{array}$} & Yes & & 1 & \multirow{2}{*}{$\mathrm{H} 12$} \\
\hline & No & 2 & If No, go to $\mathrm{H} 17$ & \\
\hline \multirow{2}{*}{$\begin{array}{l}\text { Have you ever been told by a doctor or other health worker that } \\
\text { you have raised cholesterol? }\end{array}$} & Yes & & 1 & \multirow{2}{*}{$\mathrm{H} 13 \mathrm{a}$} \\
\hline & No & 2 & If No, go to $\mathrm{H} 17$ & \\
\hline \multirow{2}{*}{ Have you been told in the past 12 months? } & Yes & & 1 & \multirow{2}{*}{$\mathrm{H} 13 \mathrm{~b}$} \\
\hline & No & & 2 & \\
\hline \multirow{2}{*}{$\begin{array}{l}\text { In the past two weeks, have you taken any oral treatment } \\
\text { (medication) for raised total cholesterol prescribed by a doctor } \\
\text { or other health worker? }\end{array}$} & Yes & & 1 & \multirow{2}{*}{$\mathrm{H} 14$} \\
\hline & No & & 2 & \\
\hline \multirow{2}{*}{ Have you ever seen a traditional healer for raised cholesterol? } & Yes & & 1 & \multirow{2}{*}{$\mathrm{H} 15$} \\
\hline & No & & 2 & \\
\hline \multirow{2}{*}{$\begin{array}{l}\text { Are you currently taking any herbal or traditional remedy for } \\
\text { your raised cholesterol? }\end{array}$} & Yes & & 1 & \multirow{2}{*}{$\mathrm{H} 16$} \\
\hline & No & & 2 & \\
\hline
\end{tabular}




\begin{tabular}{|c|c|c|c|}
\hline \multicolumn{4}{|c|}{ History of Cardiovascular Diseases } \\
\hline $\begin{array}{c}\text { Have you ever had a heart attack or chest pain from heart } \\
\text { disease (angina) or a stroke (cerebrovascular accident or } \\
\text { incident)? }\end{array}$ & Yes & 1 & $\mathrm{H} 17$ \\
\hline $\begin{array}{c}\text { Are you currently taking aspirin regularly to prevent or treat heart } \\
\text { disease? }\end{array}$ & $\begin{array}{l}\text { Yes } \\
\text { No }\end{array}$ & $\begin{array}{l}1 \\
2\end{array}$ & $\mathrm{H} 18$ \\
\hline $\begin{array}{l}\text { Are you currently taking statins } \\
\text { (Lovastatin/Simvastatin/Atorvastatin or any other statin) } \\
\text { regularly to prevent or treat heart disease? }\end{array}$ & $\begin{array}{l}\text { Yes } \\
\text { No }\end{array}$ & 1 & $\mathrm{H} 19$ \\
\hline
\end{tabular}

\begin{tabular}{|c|c|c|c|}
\hline \multicolumn{4}{|c|}{ History of Chronic Kidney Diseases } \\
\hline $\begin{array}{l}\text { Have you ever been told by a doctor or other health care } \\
\text { provider that you have kidney stones? }\end{array}$ & Yes & $\begin{array}{l}1 \\
2\end{array}$ & $\mathrm{X} 1$ \\
\hline $\begin{array}{l}\text { Have you ever been told by a doctor or other health care } \\
\text { provider that you have kidney disease, weak kidneys or low } \\
\text { kidney function? }\end{array}$ & Yes & $\begin{array}{l}1 \\
2\end{array}$ & $\mathrm{X} 2$ \\
\hline Have you ever been on dialysis or had a kidney transplant? & Yes & $\begin{array}{l}1 \\
2\end{array}$ & X3 \\
\hline
\end{tabular}

\section{Lifestyle Advice}

During the past three years, has a doctor or other health worker advised you to do any of the following? (RECORD FOR EACH)

\begin{tabular}{|c|c|c|c|}
\hline Quit using tobacco or don't start & $\begin{array}{l}\text { Yes } \\
\text { No }\end{array}$ & $\begin{array}{l}1 \\
2\end{array}$ & $\mathrm{H} 2 \mathrm{Oa}$ \\
\hline Reduce salt in your diet & $\begin{array}{l}\text { Yes } \\
\text { No }\end{array}$ & $\begin{array}{l}1 \\
2 \\
\end{array}$ & $\mathrm{H} 2 \mathrm{Ob}$ \\
\hline Eat at least five servings of fruit and/or vegetables each day & $\begin{array}{l}\text { Yes } \\
\text { No }\end{array}$ & $\begin{array}{l}1 \\
2\end{array}$ & $\mathrm{H} 2 \mathrm{Cc}$ \\
\hline Reduce fat in your diet & $\begin{array}{l}\text { Yes } \\
\text { No }\end{array}$ & $\begin{array}{l}1 \\
2 \\
\end{array}$ & $\mathrm{H} 2 \mathrm{Od}$ \\
\hline Start or do more physical activity & $\begin{array}{l}\text { Yes } \\
\text { No }\end{array}$ & $\begin{array}{l}1 \\
2 \\
\end{array}$ & $\mathrm{H} 20 \mathrm{e}$ \\
\hline Maintain a healthy body weight or lose weight & $\begin{array}{l}\text { Yes } \\
\text { No }\end{array}$ & $\begin{array}{c}1 \text { If } C 1=2 \text { go to } C X 1 \\
2\end{array}$ & $\mathrm{H} 20 \mathrm{f}$ \\
\hline
\end{tabular}




\section{Health screening}

\section{Cervical cancer}

The next question asks about cervical cancer prevention. Screening tests for cervical cancer prevention can be done in different ways, including Visual Inspection with Acetic Acid/vinegar (VIA), pap smear and Human Papillomavirus (HPV) test. VIA is an inspection of the surface of the uterine cervix after acetic acid (or vinegar) has been applied to it. For both pap smear and HPV test, a doctor or nurse uses a swab to wipe from inside your vagina, take a sample and send it to a laboratory. It is even possible that you were given the swab yourself and asked to swab the inside of your vagina. The laboratory checks for abnormal cell changes if a pap smear is done, and for the HP virus if an $\mathrm{HPV}$ test is done.

\begin{tabular}{|l|cc|c|}
\hline \multicolumn{1}{|c|}{ Question } & Response & Code \\
\hline The following questions are for women only: & Yes & 1 & \\
\hline $\begin{array}{c}\text { Have you ever had a screening test for cervical cancer, using any } \\
\text { of these methods described above? }\end{array}$ & No & 77 & CX1 \\
\hline
\end{tabular}

\begin{tabular}{|c|c|c|c|}
\hline \multicolumn{4}{|c|}{ BREAST CANCER } \\
\hline \multirow{2}{*}{$\begin{array}{l}\text { The following questions (S1-S3) are for women only: } \\
\text { Have you been shown or taught how to examine your breasts? }\end{array}$} & Yes & 1 & \multirow{2}{*}{$\mathrm{X} 4$} \\
\hline & No & 2 & \\
\hline \multirow{5}{*}{ When was the last time you had an examination of your breasts? } & 1 year or less & 1 & \multirow{5}{*}{$X 5$} \\
\hline & Between 1 and 2 years & 2 & \\
\hline & More than 2 years & 3 & \\
\hline & Never & 4 & \\
\hline & Don't know & 77 & \\
\hline \multirow{5}{*}{ When was the last time you had a mammogram? } & 1 year or less & 1 & \multirow{5}{*}{$x 6$} \\
\hline & Between 1 and 2 years & 2 & \\
\hline & More than 2 years & 3 & \\
\hline & Never & 4 & \\
\hline & Don't know & 77 & \\
\hline \multicolumn{4}{|c|}{ ORAL CANCER } \\
\hline \multirow{4}{*}{$\begin{array}{l}\text { Have you ever had lining/covering of your mouth and tongue } \\
\text { examined by a dentist or a doctor?" }\end{array}$} & Yes & 1 & \multirow{4}{*}{$X 7$} \\
\hline & No & 2 & \\
\hline & Don't know & 77 & \\
\hline & Refused & 88 & \\
\hline \multirow{5}{*}{ When did you have your most recent oral or mouth cancer exam } & 1 year or less & 1 & \multirow{5}{*}{$x 8$} \\
\hline & Between 1 and 2 years & 2 & \\
\hline & More than 2 years & 3 & \\
\hline & Never & 4 & \\
\hline & Don't know & 77 & \\
\hline
\end{tabular}




\begin{tabular}{|c|c|c|c|}
\hline \multicolumn{4}{|c|}{ Family history } \\
\hline Question & & & Code \\
\hline \multicolumn{4}{|c|}{ Have some of your family members been diagnosed with the following diseases? } \\
\hline \multirow{2}{*}{ Diabetes or raised blood sugar } & Yes & 1 & \multirow{2}{*}{$\mathrm{F} 1 \mathrm{~A}$} \\
\hline & No & 2 & \\
\hline \multirow{2}{*}{ Raised Blood pressure } & Yes & 1 & \multirow{2}{*}{$\mathrm{F} 1 \mathrm{~B}$} \\
\hline & No & 2 & \\
\hline \multirow{2}{*}{ Stroke } & Yes & 1 & \multirow{2}{*}{$\mathrm{F} 1 \mathrm{C}$} \\
\hline & No & 2 & \\
\hline \multirow{2}{*}{ Cancer or malignant tumor } & Yes & 1 & \multirow{2}{*}{ F1D } \\
\hline & No & 2 & \\
\hline \multirow{2}{*}{ Raised Cholesterol } & Yes & 1 & \multirow{2}{*}{ F1E } \\
\hline & No & 2 & \\
\hline \multirow{2}{*}{$\begin{array}{l}\text { Early Heart attack (below age } 55 \text { for men and below age } 65 \text { for } \\
\text { women) }\end{array}$} & Yes & 1 & \multirow{2}{*}{ F1f } \\
\hline & No & 2 & \\
\hline \multirow{2}{*}{ Chronic Kidney Diseases } & Yes & 1 & \multirow{2}{*}{ F1g } \\
\hline & No & 2 & \\
\hline
\end{tabular}


'Mental health / Suicide

Over the last 2 weeks, how often have you been bothered by any of the following problems?

The next questions ask about thoughts, plans, and attempts of suicide. Please answer the questions even if no one usually talks about these issues.

\begin{tabular}{|c|c|c|c|}
\hline Question & Response & & Code \\
\hline $\begin{array}{c}\text { During the past } 2 \text { weeks, have you had little interest or } \\
\text { pleasure in doing things }\end{array}$ & $\begin{array}{r}\text { Not at all } \\
\text { Several days } \\
\text { More than half days } \\
\text { Nearly every day }\end{array}$ & $\begin{array}{l}0 \\
1 \\
2 \\
3\end{array}$ & MHX1 \\
\hline $\begin{array}{l}\text { During the past } 2 \text { weeks, have you felt down, } \\
\text { depressed, or hopeless }\end{array}$ & $\begin{array}{r}\text { Not at all } \\
\text { Several days } \\
\text { More than half days } \\
\text { Nearly every day }\end{array}$ & $\begin{array}{l}0 \\
1 \\
2 \\
3\end{array}$ & $\mathrm{MHX} 2$ \\
\hline $\begin{array}{l}\text { During the past } 2 \text { weeks, have you had trouble falling } \\
\text { or staying asleep, or sleeping too much }\end{array}$ & $\begin{array}{r}\text { Not at all } \\
\text { Several days } \\
\text { More than half days } \\
\text { Nearly every day }\end{array}$ & $\begin{array}{l}0 \\
1 \\
2 \\
3\end{array}$ & $\mathrm{MHX3}$ \\
\hline $\begin{array}{l}\text { During the past } 2 \text { weeks, are you overeating or have a } \\
\text { poor appetite }\end{array}$ & $\begin{array}{r}\text { Not at all } \\
\text { Several days } \\
\text { More than half days } \\
\text { Nearly every day }\end{array}$ & $\begin{array}{l}0 \\
1 \\
2 \\
3\end{array}$ & $\mathrm{MHX5}$ \\
\hline $\begin{array}{c}\text { During the past } 2 \text { weeks, are you feeling bad about } \\
\text { yourself - or that you are a failure or have let yourself } \\
\text { or your family down }\end{array}$ & $\begin{array}{r}\text { Not at all } \\
\text { Several days } \\
\text { More than half days } \\
\text { Nearly every day }\end{array}$ & $\begin{array}{l}0 \\
1 \\
2 \\
3\end{array}$ & MHX6 \\
\hline $\begin{array}{c}\text { During the past } 2 \text { weeks, are you having trouble } \\
\text { concentrating on things, such as reading the } \\
\text { newspaper or watching television }\end{array}$ & $\begin{array}{r}\text { Not at all } \\
\text { Several days } \\
\text { More than half days } \\
\text { Nearly every day }\end{array}$ & $\begin{array}{l}0 \\
1 \\
2 \\
3\end{array}$ & MHX7 \\
\hline $\begin{array}{c}\text { During the past } 2 \text { weeks, have you had thoughts that } \\
\text { you would be better off dead or of hurting yourself in } \\
\text { some way }\end{array}$ & $\begin{array}{r}\text { Not at all } \\
\text { Several days } \\
\text { More than half days } \\
\text { Nearly every day }\end{array}$ & $\begin{array}{l}0 \\
1 \\
2 \\
3\end{array}$ & $\mathrm{MHX9}$ \\
\hline $\begin{array}{l}\text { During the past } 12 \text { months, have you seriously } \\
\text { considered attempting suicide? }\end{array}$ & $\begin{array}{r}\text { Yes } \\
\text { No } \\
\text { Refused }\end{array}$ & $\begin{array}{c}1 \\
\text { 2If No, go to } M H 3 \\
88\end{array}$ & $\mathrm{MH} 1$ \\
\hline Did you seek professional help for these thoughts? & $\begin{array}{r}\text { Yes } \\
\text { No } \\
\text { Refused }\end{array}$ & $\begin{array}{c}1 \\
2 \\
88\end{array}$ & $\mathrm{MH} 2$ \\
\hline $\begin{array}{l}\text { During the past } 12 \text { months, have you made a plan } \\
\text { about how you would attempt suicide? }\end{array}$ & $\begin{array}{r}\text { Yes } \\
\text { No }\end{array}$ & $\begin{array}{l}1 \\
2\end{array}$ & $\mathrm{MH} 3$ \\
\hline
\end{tabular}




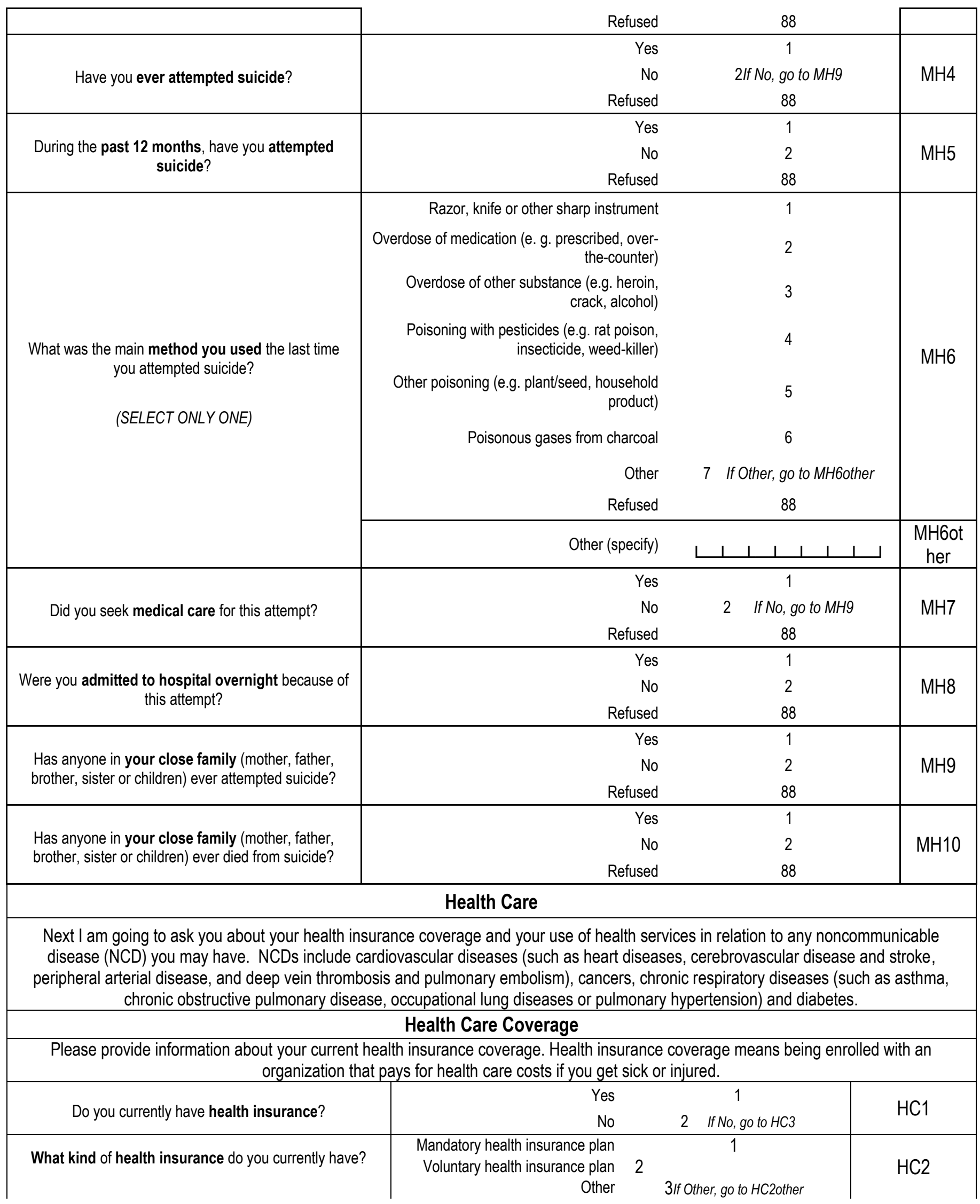




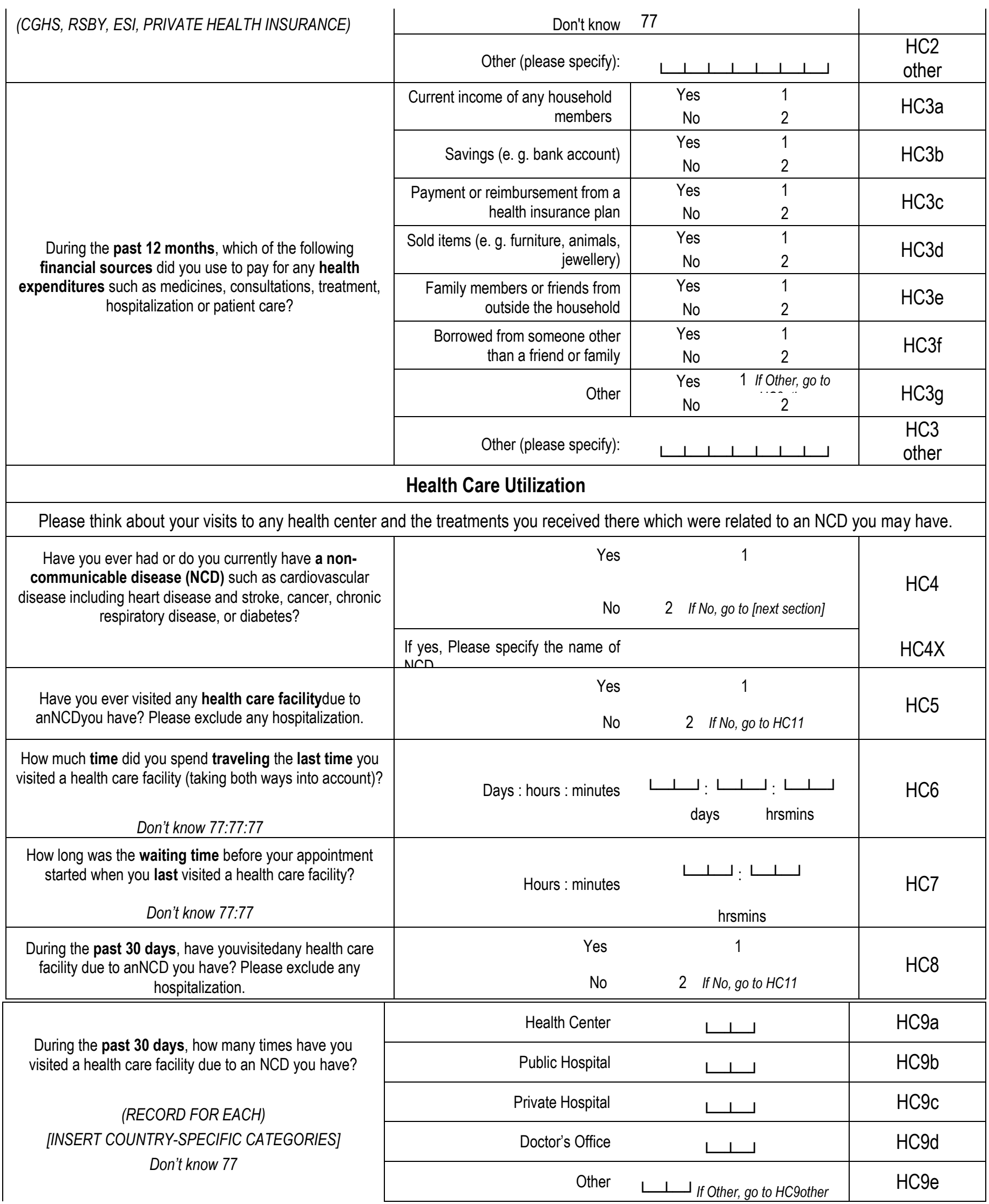




\begin{tabular}{|c|c|c|c|}
\hline & Other (please specify): & $\begin{array}{lllllll} & \perp & 1 & 1 & 1 & 1 & \perp\end{array}$ & HC9otehr \\
\hline \multirow{3}{*}{$\begin{array}{c}\text { During the past } 30 \text { days, taking all your visits to a health } \\
\text { care facility due to an NCD into account, how much did } \\
\text { you pay yourself for these visits in total? }\end{array}$} & Health care provider's fees & 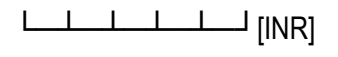 & $\mathrm{HC} 10 \mathrm{a}$ \\
\hline & Medicines & 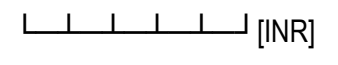 & $\mathrm{HC10b}$ \\
\hline & Tests & 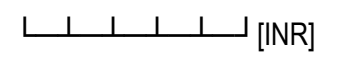 & $\mathrm{HC10c}$ \\
\hline \multirow{3}{*}{$\begin{array}{c}\text { (RECORD FOR EACH OR PUT TOTAL AMOUNT) } \\
\text { Don't know } 77777\end{array}$} & Transport & 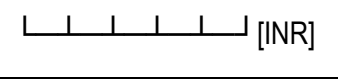 & HC10d \\
\hline & Other & 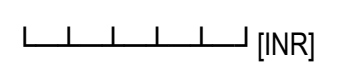 & HC10e \\
\hline & OR Total Amount & 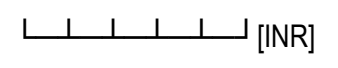 & HC10f \\
\hline $\begin{array}{c}\text { During the past } 30 \text { days, how much did you pay } \\
\text { yourself for health care not related to any visit of a health } \\
\text { care facility or hospital, such as routine medication? } \\
\text { Don't know } 77777\end{array}$ & Amount & 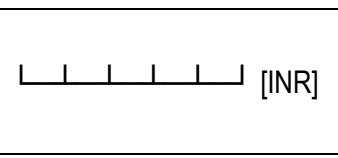 & HC11 \\
\hline $\begin{array}{l}\text { During the past } 12 \text { months, have you been hospitalized } \\
\text { due to an NCD? }\end{array}$ & $\begin{array}{r}\text { Yes } \\
\text { No }\end{array}$ & $\begin{array}{c}1 \\
2 \quad \text { If No, go to } \mathrm{HC} 15 \\
\end{array}$ & $\mathrm{HC} 12$ \\
\hline $\begin{array}{c}\text { During the past } 12 \text { months, how many days have you } \\
\text { been hospitalized due to an NCD? } \\
\text { Don't know } 777\end{array}$ & Number of days & 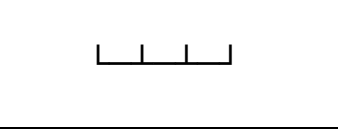 & $\mathrm{HC} 13$ \\
\hline \multirow{6}{*}{$\begin{array}{l}\text { During the past } 12 \text { months, taking all your visits to a } \\
\text { hospital due to an NCD into account, how much did you } \\
\text { pay yourself for these visits in total? }\end{array}$} & Health care provider's fees & $\begin{array}{lllll}\perp & \perp & \perp & \perp & \perp\end{array}$ & $\mathrm{HC} 14 \mathrm{a}$ \\
\hline & Medicines & 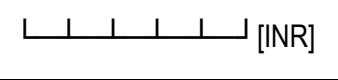 & $\mathrm{HC} 14 \mathrm{~b}$ \\
\hline & Tests & 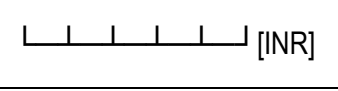 & $\mathrm{HC} 14 \mathrm{c}$ \\
\hline & Transport & $\left.\begin{array}{llllll}\perp & \perp & \perp & \perp & \perp & \perp\end{array}\right]$ & $\mathrm{HC} 14 \mathrm{~d}$ \\
\hline & Other & $\left.\begin{array}{lllll}\perp & \perp & \perp & \perp & \perp\end{array}\right]$ & $\mathrm{HC} 14 \mathrm{e}$ \\
\hline & OR Total Amount & 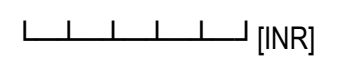 & $\mathrm{HC} 14 \mathrm{f}$ \\
\hline \multicolumn{4}{|c|}{ Home Care } \\
\hline \multicolumn{4}{|c|}{ Please think about home care from family membersand/or friends because of an NCD you have. } \\
\hline $\begin{array}{c}\text { During the past } 30 \text { days, has a family member or friend } \\
\text { provided care for you at home due to your NCD? }\end{array}$ & $\begin{array}{r}\text { Yes } \\
\text { No }\end{array}$ & $\begin{array}{c}1 \\
2 \quad \text { If No, go to } \mathrm{HC} 17 \\
\end{array}$ & HC15 \\
\hline $\begin{array}{c}\text { During the past } 30 \text { days, how many hours per week has } \\
\text { this person/have these people provided care for you? } \\
\text { Don't know } 777\end{array}$ & Hours per week & $\begin{array}{c}1 \perp \perp 1 \\
\text { hrs }\end{array}$ & $\mathrm{HC16}$ \\
\hline \multicolumn{4}{|c|}{ Loss of Productivity } \\
\hline \multicolumn{4}{|c|}{ Please think about the time you couldn't do your usual activity (for example, work, work at home, study) because of an NCD you have. } \\
\hline $\begin{array}{l}\text { During the past } 30 \text { days, have you missed any time of } \\
\text { your usual activity (work, work at home, study) due to an } \\
\text { NCD? }\end{array}$ & $\begin{array}{l}\text { Yes } \\
\text { No }\end{array}$ & $\begin{array}{c}1 \\
2 \text { If No, go to [next section] }\end{array}$ & $\mathrm{HC17}$ \\
\hline $\begin{array}{c}\text { During the past } 30 \text { days, how many days of your usual } \\
\text { activity have you missed due to an NCD? } \\
\text { Don't know } 77\end{array}$ & Number of days & days & HC18 \\
\hline
\end{tabular}




\begin{tabular}{|c|c|c|c|}
\hline \multicolumn{4}{|c|}{ Physical Measurements } \\
\hline \multicolumn{4}{|c|}{ Blood Pressure } \\
\hline Question & \multicolumn{2}{|c|}{ Response } & \multirow{2}{*}{$\frac{\text { Code }}{\text { M1 }}$} \\
\hline Interviewer ID & & 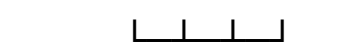 & \\
\hline Device ID for blood pressure & \multicolumn{2}{|r|}{ 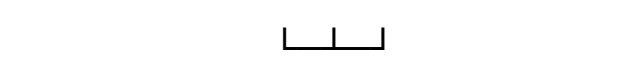 } & M2 \\
\hline \multirow{2}{*}{ Reading 1} & Systolic ( mmHg) & 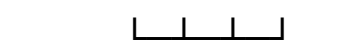 & $\mathrm{M} 4 \mathrm{a}$ \\
\hline & Diastolic (mmHg) & 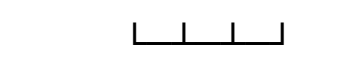 & M4b \\
\hline \multirow{2}{*}{ Reading 2} & Systolic ( mmHg) & 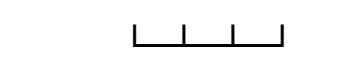 & M5a \\
\hline & Diastolic (mmHg) & 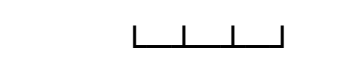 & M5b \\
\hline \multirow{2}{*}{ Reading 3} & Systolic ( mmHg) & 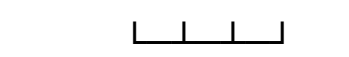 & M6a \\
\hline & Diastolic (mmHg) & 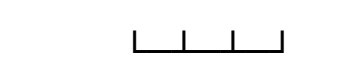 & M6b \\
\hline $\begin{array}{l}\text { During the past two weeks, have you been treated for raised } \\
\text { blood pressure with drugs (medication) prescribed by a doctor } \\
\text { or other health worker? }\end{array}$ & $\begin{array}{l}\text { Yes } \\
\text { No }\end{array}$ & $\begin{array}{l}1 \\
2\end{array}$ & M7 \\
\hline \multicolumn{4}{|c|}{ Height and Weight } \\
\hline Interviewer ID & & 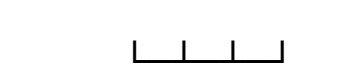 & M9 \\
\hline \multirow{2}{*}{ Device IDs for height and weight } & Height & $\downarrow$ & M10a \\
\hline & Weight & $\downarrow$ & M10b \\
\hline Height & in Centimetres (cm) & 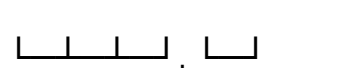 & M11 \\
\hline $\begin{array}{l}\text { Weight } \\
\text { If too large for scale } 666.6\end{array}$ & in Kilograms (kg) & 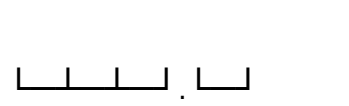 & M12 \\
\hline \multicolumn{4}{|c|}{ Waist } \\
\hline Device ID for waist & & & M13 \\
\hline Waist circumference & in Centimetres (cm) & 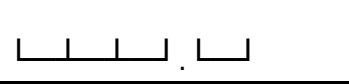 & M14 \\
\hline \multicolumn{4}{|c|}{ Hip Circumference and Heart Rate } \\
\hline Hip circumference & in Centimeters (cm) & 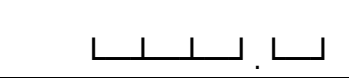 & M15 \\
\hline \multicolumn{4}{|c|}{ Heart Rate } \\
\hline Reading 1 & Beats per minute & 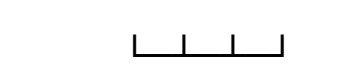 & M16a \\
\hline Reading 2 & Beats per minute & 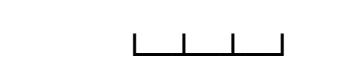 & M16b \\
\hline
\end{tabular}




\begin{tabular}{|c|c|c|c|}
\hline Reading 3 & Beats per minute & 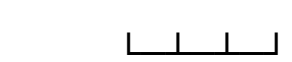 & M16c \\
\hline \multicolumn{4}{|c|}{ Skin Fold Thickness } \\
\hline Triceps & in millimetres (mm) & 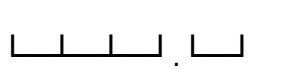 & MR2X \\
\hline Suprailiac & in millimetres (mm) & 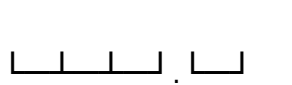 & MR3X \\
\hline Subscapular & in millimetres (mm) & 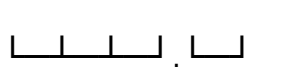 & MR4X \\
\hline
\end{tabular}

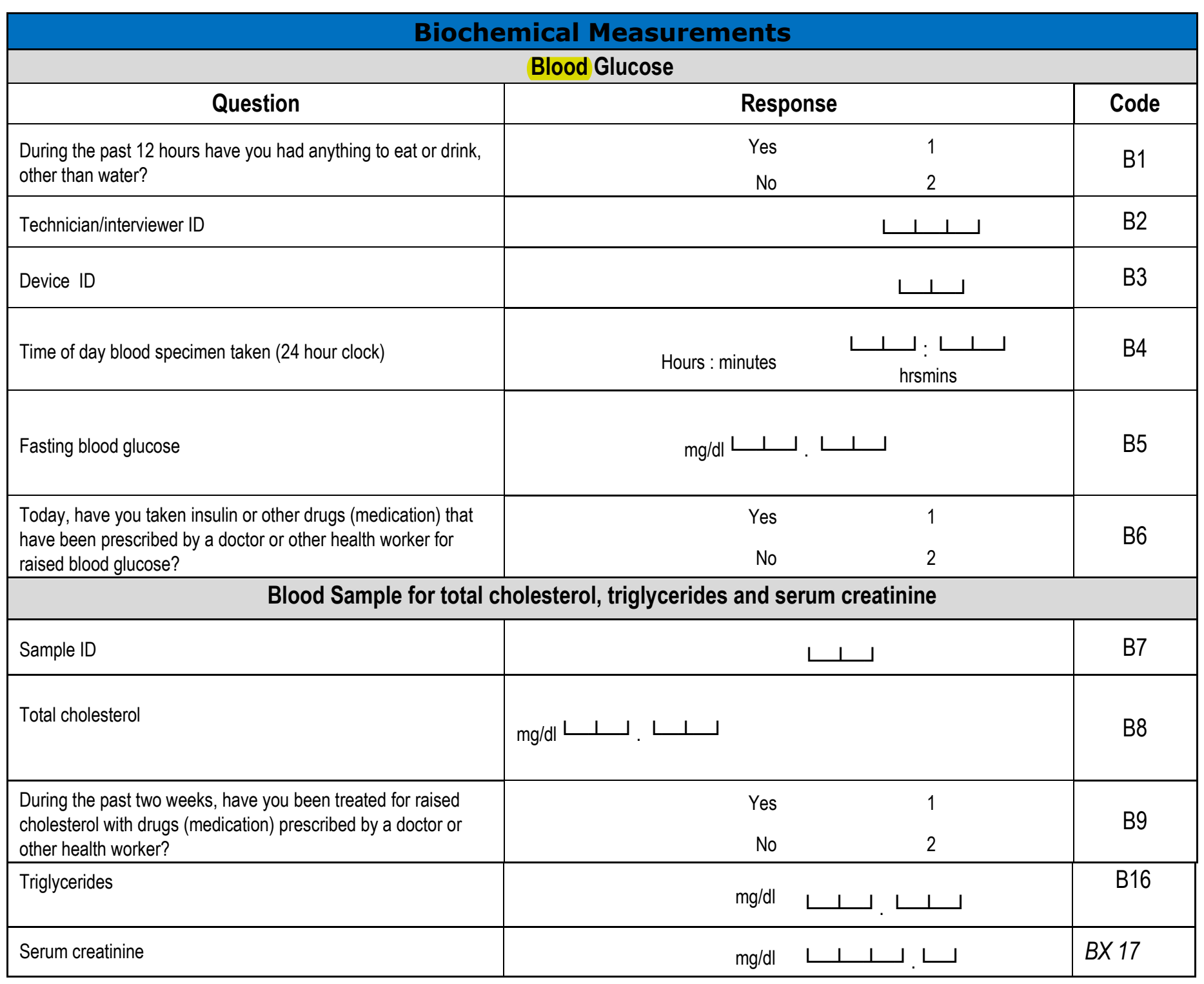

Urinary sodium, albumin and creatinine 
Technician ID

Sample ID

Time of day urine sample taken (24 hour clock)

Urinary sodium

Urinary creatinine

Urinary albumin

Albumin creatinine ratio

Serum Cystatin C

Serum High Density Cholesterol

Serum Low Density Cholesterol
No

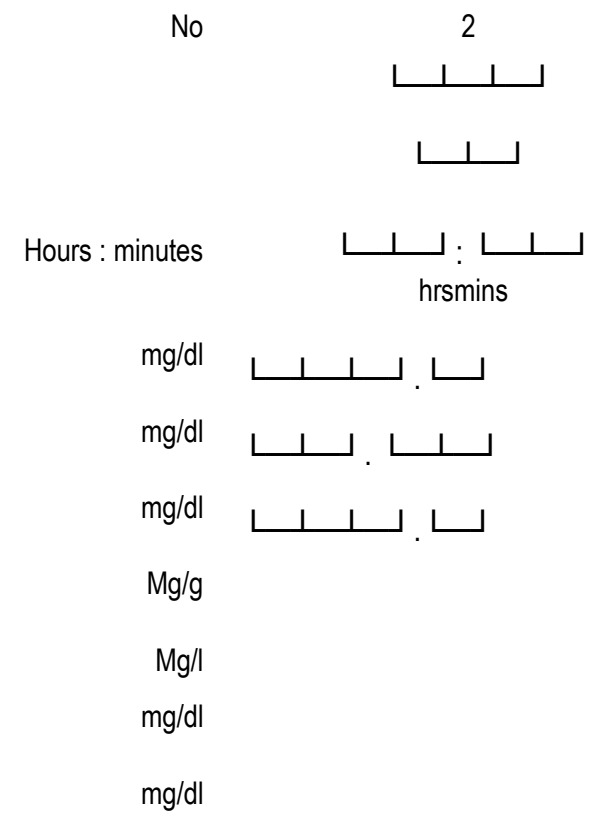

B11

B12

B13

B14

B15

BX16

BX18

BX 19

BX 20

BX 21 